Research Article

\title{
Improvement of the Communication between RFID Sensor Network Devices Used to Control and Monitor a Building
}

\author{
Abdelhamid Bou-El-Harmel $\mathbb{D}$, Ali Benbassou, and Jamal Belkadid \\ Laboratory of Innovative Technologies Eq. CEM/Telecoms Sidi Mohamed Ben Abdullah University, High School of Technology, \\ Road IMOUZZER, BP $2427 \mathrm{Fez}$, Morocco \\ Correspondence should be addressed to Abdelhamid Bou-El-Harmel; abdelhamid.bouelharmel@usmba.ac.ma
}

Received 24 July 2020; Revised 5 January 2021; Accepted 10 January 2021; Published 29 January 2021

Academic Editor: Giorgio Pennazza

Copyright (c) 2021 Abdelhamid Bou-El-Harmel et al. This is an open access article distributed under the Creative Commons Attribution License, which permits unrestricted use, distribution, and reproduction in any medium, provided the original work is properly cited.

\begin{abstract}
In the RFID sensor network (RSN), the devices communicate with each other by RF waves using the antennas through a propagation channel. A poor communication between these devices results in either a significant economic loss or security threats. The communication problems can have several origins depending on the type of antenna used and the nature of the propagation channel. In this work, our objective is to limit the communication problems between the nodes of this network that are linked to the characteristics of an indoor propagation channel. The goal is to predict the channel characteristics using the 3D ray tracing method in order to select the appropriate transmission parameters such as transmission power and duration of a symbol. To achieve this, we have modeled a building that is sectioned as a propagation channel where network devices are deployed for control and monitoring. The communication was made at $915 \mathrm{MHz}$ using the quasi-isotropic 3D cubic antenna that we designed as well as a conventional dipole antenna in order to compare the results. We have found that the use of the 3D cubic antenna gives several advantages to the RFID sensor network compared to the most commonly used conventional dipole antenna, such as a transmission power of $0 \mathrm{dBm}$ which automatically leads to an increase in the lifetime of the devices, as well as a minimum symbol duration of around $219.78 \mathrm{~ns}$ which gives a high bit rate.
\end{abstract}

\section{Introduction}

The RSN network is a new research field for industrialists and academics. It combines the properties of RFID technology to WSN technology and vice versa $[1,2]$. This association makes it possible to extend the operational and functional capabilities of the devices in order to respond to the needs of the applications.

In RSN applications, the devices communicate with each other by RF waves using the antennas through a propagation channel. A poor communication between these devices results in either a significant economic loss or security threats. The communication problems can have several origins depending on the type of antenna used and the nature of the propagation channel.

The antennas are fundamental and essential elements in communication between devices of the RSN network. Each antenna has different radiation characteristics. The most commonly used antennas are dipoles and monopoles [3, 4]. The latter produce radiation in the form of a doughnut with zero field strength areas along their axis. In these areas, poor communication occurs between network nodes.

In order to ensure reliable communication between RSN nodes at the antennas level, we have designed in previous work new antennas in 3D form that produce quasiisotropic radiation, such as the cubic $[5,6]$ and spherical [7] antennas.

Regarding the propagation channel, it corresponds to the environment traversed by the RF waves during the transmission of information between the transmitting and receiving antennas of the nodes of the RSN network. Most RSN applications are deployed inside buildings, which constitutes a multipath propagation channel. These multiple paths can generate destructive interference at the receiving node which produces a fading of the received signal. Thus, the long propagation delays between the different paths and their variation 


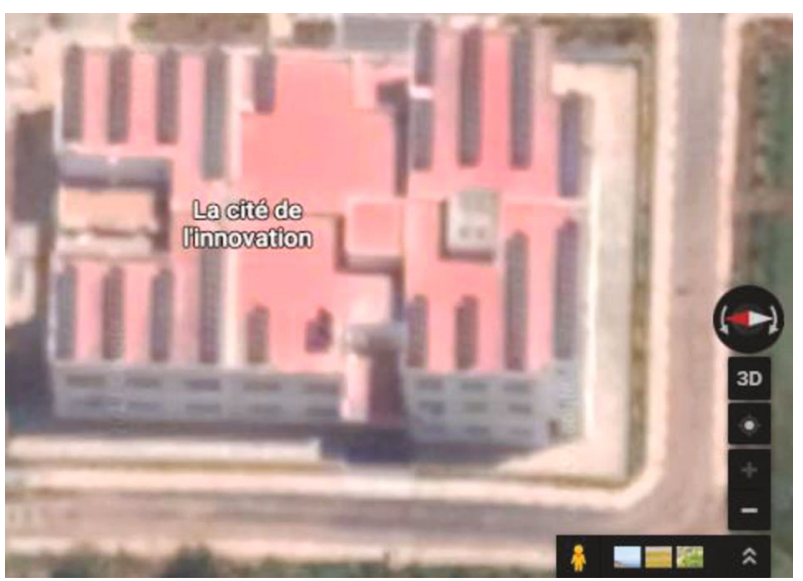

Figure 1: A view of the building taken by Google Maps.

with time can cause intersymbol interference (ISI) and symbol estimation errors (SEE), respectively. All of these problems contribute to poor RF transmission between network nodes.

A good transmission then requires a good choice of transmission parameters such as the transmission power $P_{t}$ and the symbol duration $T_{s}$. These parameters are directly linked to the characteristics of the propagation channel, i.e., received power $P_{r}$, coherence band $B_{c}$, and coherence time $T_{c}$.

In previous work [8], we have studied the effects of the orientation of two different antennas on the characteristics of the multipath propagation channel in a typical indoor environment. The propagation channel characteristics are compared in terms of received power level $\left(P_{r}\right)$ and delay spread $\left(\tau_{\mathrm{RMS}}\right)$ for the $3 \mathrm{D}$ cubic antenna and the conventional dipole in the LOS, NLOS, and OLOS scenarios.

In this work, our objective is to predict the appropriate transmission parameters $\left(P_{t}\right.$ and $\left.T_{s}\right)$ using our $3 \mathrm{D}$ cubic antenna and the conventional dipole antenna as transmitting and receiving antenna of the RSN network and to compare the results obtained. For this purpose, we will in "Environment, Location, and Antennas" model the indoor environment (building) that has been selected as the propagation channel where the RSN network will be deployed, as well as specify the location of the nodes and determine the antennas used with their radiation characteristics. In "3D Ray Tracing Method", we will present the 3D ray tracing method. In "Results and Analysis", we will present the predicted propagation channel characteristics as well as the appropriate transmission parameters with a comparison between the results obtained.

\section{Environment, Location, and Antennas}

2.1. Selected Environment. The selected environment as the propagation channel where the RSN network is deployed is the one we have already modeled in the work [8]. We remind that this environment is a building within our university [9] (see Figure 1).

The complete modeling of this two-floor building is very complex. We were only interested in modeling the north wing of the first floor (see Figure 2) where our laboratory is

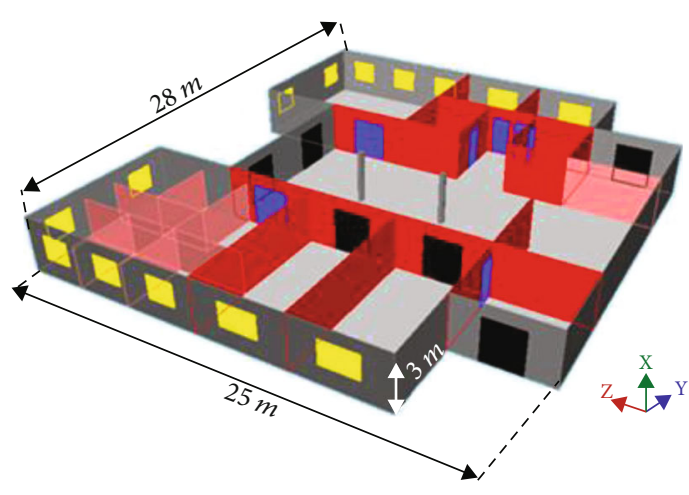

FIGURE 2: First floor of the north wing of the building in 3D.

TABLE 1: Dielectric constants of materials.

\begin{tabular}{lcc}
\hline Types & \multicolumn{3}{c}{$\boldsymbol{\varepsilon}_{r}=\boldsymbol{\varepsilon}_{r}^{\prime}+\mathbf{j} \boldsymbol{\epsilon}_{r}^{\prime}$} & $\varepsilon_{r}^{\prime}$ \\
\hline Reinforced-concrete wall & $\varepsilon_{r}^{\prime}$ & -1.2 \\
Brick wall & 5.7 & -0.2 \\
Glass partition & 5 & 0 \\
Iron door & 1 & 0 \\
Wood door & 3 & 0 \\
Glass window & 5 & 0 \\
Ground & 10 & -1.2 \\
Roof & 10 & -1.2 \\
\hline
\end{tabular}

located. This floor consists of reinforced-concrete and brick walls, glass partitions, iron and wooden doors, and glass windows.

All the constituents of our environment are considered homogeneous and having complex dielectric constants (see Table 1) obtained from a free space method using transmission measurements at normal incidence [10]. Obstacles such as cupboards, machines, and desks are not modeled because of the complexity of their design.

2.2. Location of RSN Nodes. The RSN network is used for control and monitoring of the building with a star topology, as shown in Figure 3. The terminal nodes are deployed and fixed above the doors and windows with a height from the ground of $2.5 \mathrm{~m}$. This position is most optimal for integrating several types of sensors (motion sensor, temperature sensor, gas sensor, camera, etc.) with the magnetic contact sensor which is used to monitor the opening and closing of doors and windows. All detected information is transmitted to the coordinator node which is located in the middle of the corridor with a height above the ground of $2.5 \mathrm{~m}$. In the star topology, the network is controlled by the coordinator node, so we choose it as a transmitter $(T x)$ and the terminal nodes as receivers $(R x)$.

2.3. Used Antennas. In this study, we use, for RF communication between the nodes of the network, the 3D cubic antenna that we designed and the conventional half-wave dipole antenna. We remind that the $3 \mathrm{D}$ cubic antenna produces a 
quasi-isotropic radiation pattern with a maximum gain of $1.29 \mathrm{dBi}$ and circular polarization; on the other hand, the dipole antenna produces a radiation in the form of a doughnut with a maximum gain of $2.14 \mathrm{dBi}$ and a polarization linear. The position of these two antennas relative to the environment is shown in Figure 4.

During communication, the frequency and power of transmission used are, respectively $f=915 \mathrm{MHz}$ (the resonant frequency of the two antennas) and $P_{t}=0 \mathrm{dBm}$ (the minimum transmission power of the RSN devices).

2.4. 3D Ray Tracing Method. The 3D ray tracing method is an asymptotic method based on the following:

(i) The Geometric Optics (GO) method [11] to describe the direct, reflected and transmitted fields by the concept of rays, as shown in Figure 5(a)

(ii) The Uniform Diffraction Theory (UDT) [12] to describe the diffracted fields by the concept of rays (see Figure 5(b)).

To identify the rays (paths) that propagate between the antennas, the RT-3D method uses the image method and the folding method [13]. The implementation of the RT-3D method is very complex but offers a complete description of the received waves [14]. Once all propagation rays are determined, the received power in $\mathrm{dB}$ is calculated by [15]:

$$
P_{r}[d B]=10 \log \left(\frac{\left|E_{t o t}\right|^{2}}{\eta_{0}} A\right),
$$

where

(i) $\eta_{0}=\sqrt{\mu_{0} / \epsilon_{0}}=120 \pi$ is the intrinsic impedance of air with $\mu_{0}$ and $\epsilon_{0}$ being the permeability and permittivity of the vacuum, respectively

(ii) $A=\left(\lambda^{2} G_{r} / 4 \pi\right)$ is the effective area of the receiving antenna with $\lambda$ being the wavelength and $G_{r}$ the gain of the receiving antenna

(iii) $E_{\text {tot }}=\sum_{i} E_{i}$ is the combination of the direct field between the $T x$ node and the $R x$ node, the fields reflected by obstacles, the fields transmitted through obstacles, and the fields diffracted by the edges of obstacles. With

$$
E_{i}=\frac{E_{0} f_{t} f_{r} e^{-j k d_{i}}}{d_{i}} \prod_{a=1}^{n} \mathrm{R}_{a} \prod_{b=1}^{m} T_{b} \prod_{c=1}^{l} D_{c}
$$

where

(i) $E_{0}=P_{t} G_{e} / 4 \pi$ is the reference field

(ii) $f_{t}$ and $f_{r}$ are the characteristic functions of the radiation in the ray direction between transmitting and receiving antennas, respectively

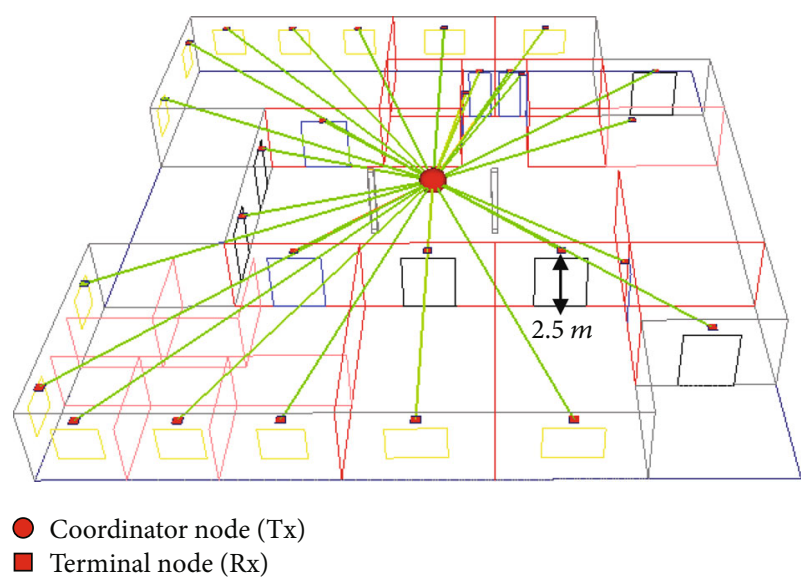

FIgURE 3: Location of the nodes of the RSN network according to the star topology.

(iii) $k=2 \pi / \lambda$ is the constant of propagation

(iv) $d_{i}$ is the length of path $i$

(v) $n, \mathrm{~m}$, and $l$ are the total numbers of reflections, transmissions, and diffractions, respectively

(vi) $R_{a}, T_{b}$, and $D_{c}$ are the reflection coefficient of the $a^{\text {th }}$ reflection, the transmission coefficient for the $b^{\text {th }}$ transmission, and the diffraction coefficient for the $c^{\text {th }}$ diffraction, respectively.

During the simulation, the maximum number of reflections, transmissions, and diffractions is in the order of 5. The receivers of the RSN node are capable of achieving a sensitivity between $-92 \mathrm{dBm}$ and $-107.5 \mathrm{dBm}[16,17]$. Therefore, we will choose the $-92 \mathrm{dBm}$ value as the sensitivity threshold of the $R x$.

\section{Results and Analysis}

3.1. Received Power. After the simulations, Figure 6 presents the prediction results of the received power $P_{r}[\mathrm{dBm}]$ at each $R x$ using the two antennas ((a) 3D cubic and (b) dipole). According to the results, we observe that the received power $P_{r}$ varies randomly as a function of the distance between $T x$ and each $R x$, which is due to the different paths received at each $R x$.

When using the 3D cubic antenna, we have obtained a minimum power value of $-77.35 \mathrm{dBm}$ at distance $14.03 \mathrm{~m}$. However, during the use of the conventional dipole antenna, the minimum value of the power is $-74.18 \mathrm{dBm}$ at the distance of $14.44 \mathrm{~m}$.

In this study, we have positioned the 3D cubic antenna of the RSN nodes in the same way throughout the environment. The orientation of the antennas relative to each other changes the values of the power $P_{r}$ as we have found in the work [8]. Table 2 summarizes the maximum differences between the levels of the power $P_{r}$ obtained during all the orientations of the 3D cubic and dipole antennas in the LOS, NLOS, and OLOS scenarios. 

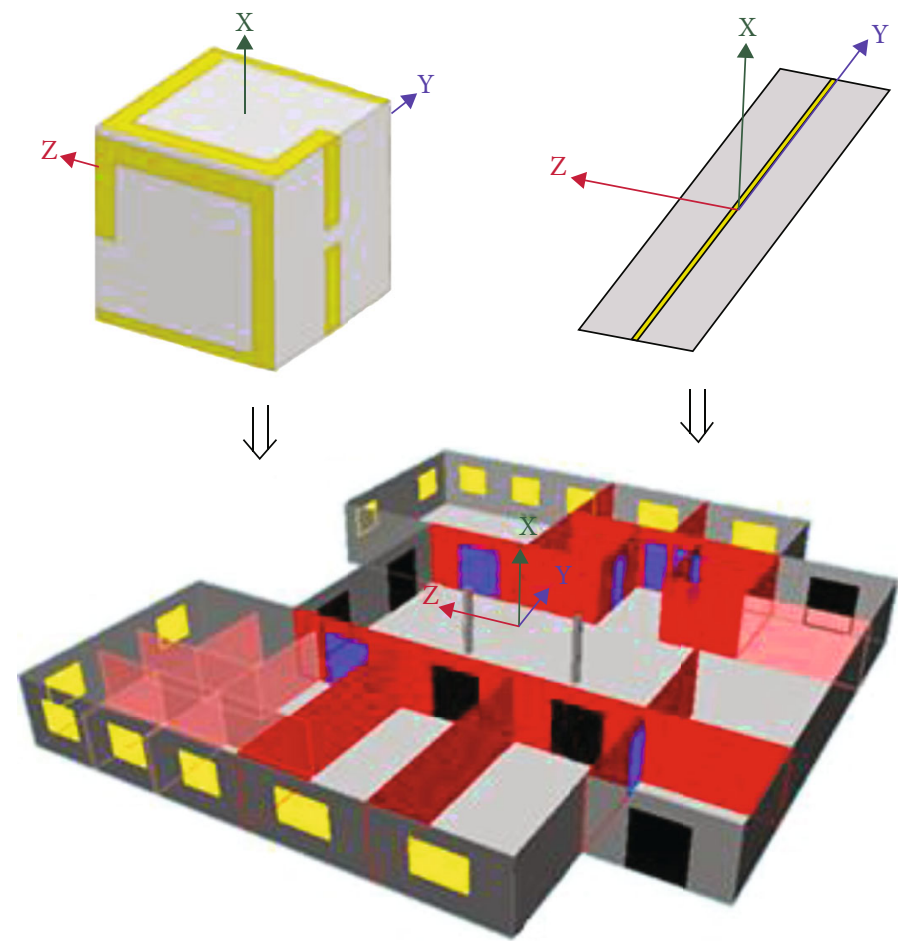

Figure 4: Position of the antennas in relation to the environment.

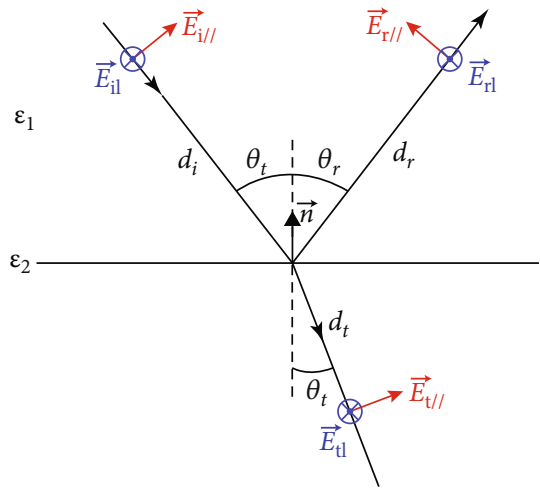

(a)

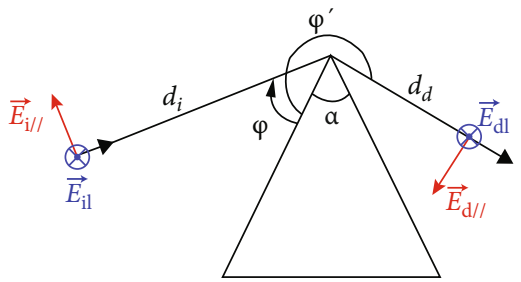

(b)

FIgURE 5: (a) Reflection and transmission of an incident wave and (b) diffraction of an incident wave.

As shown in Table 2, the two maximum power differences obtained when orienting the $3 \mathrm{D}$ cubic antenna and the dipole antenna are $6.85 \mathrm{dBm}$ and $27.97 \mathrm{dBm}$, respectively. Therefore, the minimum value of the power obtained when using the $3 \mathrm{D}$ cubic antenna becomes $-84.2 \mathrm{dBm}$ and when using the dipole antenna becomes $-102.15 \mathrm{dBm}$.

As regards the fight against the fading of the received signal, the minimum value of the power $P_{r}$ must be greater than the sensitivity threshold 4 of the $R x(-92 \mathrm{dBm})$. This implies that the value of the transmission power $P_{t}(0 \mathrm{dBm})$ chosen at the outset is satisfactory to ensure reliable RF transmission between the nodes of the RSN network using the 3D cubic antenna. On the contrary, the power $P_{t}$ must be increased when using the dipole antenna, which will decrease the lifespan of the devices.

3.2. Coherence Band. The coherence band $B_{c}$ is obtained from the maximum delay $T_{m}$ by the following relation:

$$
B_{c}=\frac{1}{T_{m}},
$$

with $T_{m}$ being the delay between the first and last pulse of the impulse response (IR) of each channel, as shown in Figure 7 which represents three predicted IRs at the level of three $R x$ using the $3 \mathrm{D}$ cubic antenna. 


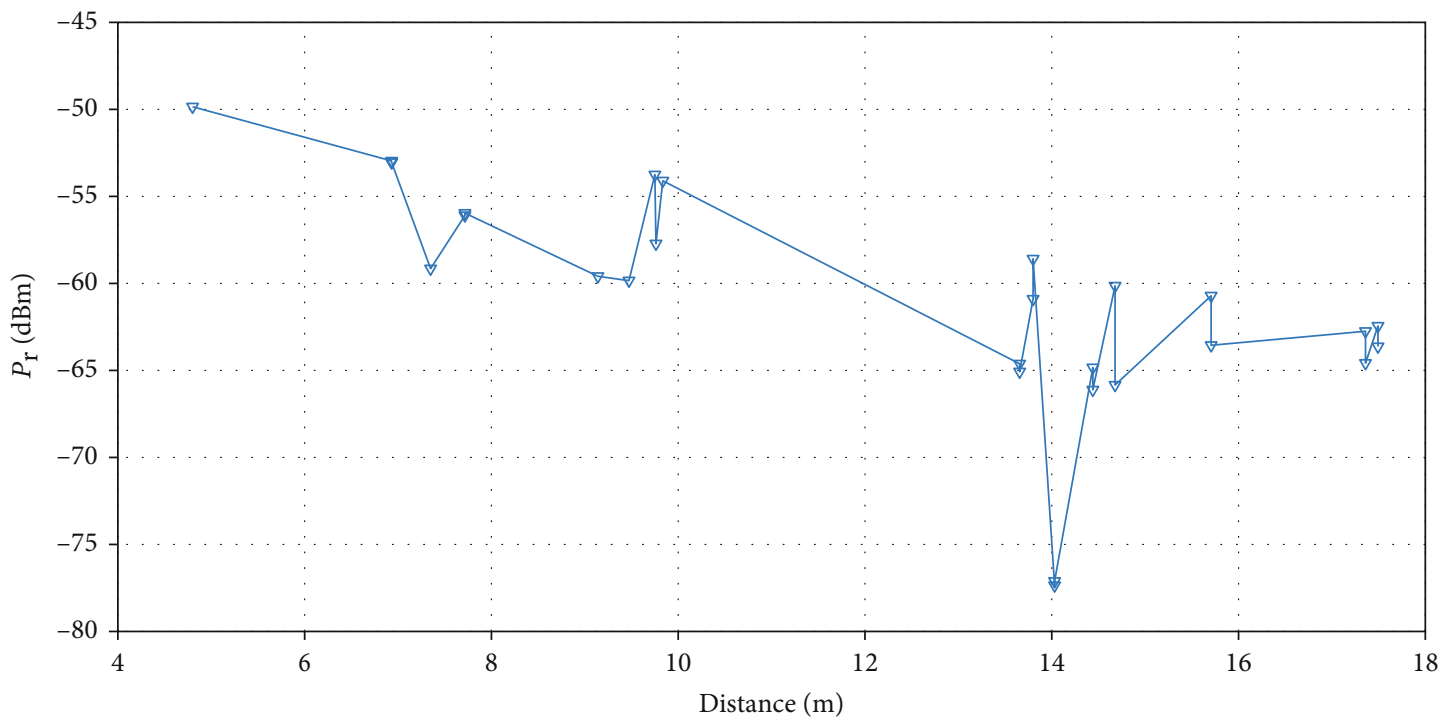

(a)

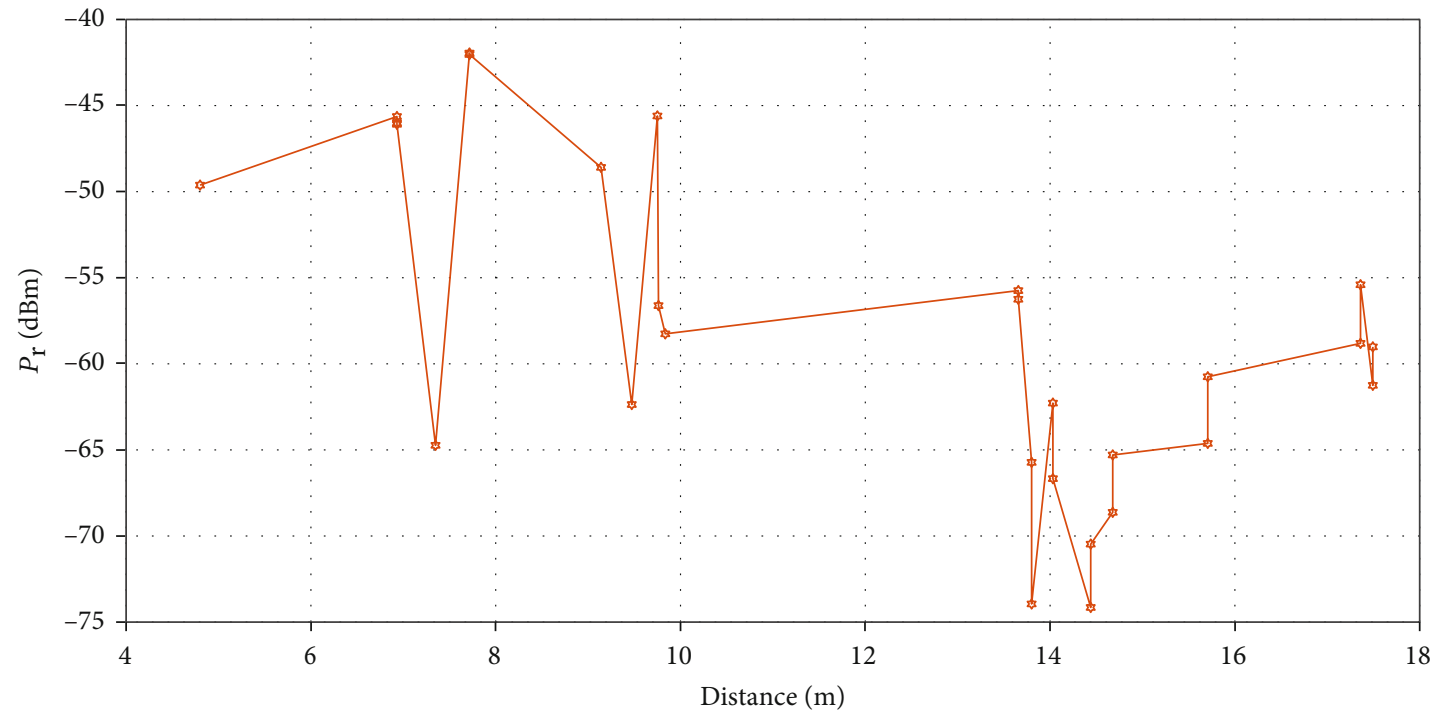

(b)

Figure 6: $P_{r}$ predicted as a function of the distance between $T x$ and each $R x$ when using (a) the 3D cubic antenna and (d) the dipole antenna.

Table 2: Maximum differences between the levels of the $P_{r}$ during the orientations of the $3 \mathrm{D}$ cubic and dipole antennas [8].

\begin{tabular}{lcc}
\hline Scenarios & 3D cubic antenna & Dipole antenna \\
\hline LOS & $6.85 \mathrm{dBm}$ & $27.81 \mathrm{dBm}$ \\
NLOS & $6.07 \mathrm{dBm}$ & $25.08 \mathrm{dBm}$ \\
OLOS & $4.6 \mathrm{dBm}$ & $27.97 \mathrm{dBm}$ \\
\hline
\end{tabular}

In the case where the transmission power $P_{t}$ is insufficient, the increase of this one leads to an increase in the number of paths received, which implies an increase in the values of the delay $T_{m}$. Therefore, we will use the delay dispersion $\tau_{\text {RMS }}$ which takes into account the importance of the paths in order to calculate the coherence band:

$$
\tau_{\mathrm{RMS}}=\sqrt{\frac{\sum_{i} P_{i} \tau_{i}^{2}}{\sum_{i} P_{i}}-\left[\frac{\sum_{i} P_{i} \tau_{i}}{\sum_{i} P_{i}}\right]^{2}} .
$$

The relationship between the coherence band $B_{c}$ and the delay spread $\tau_{\text {RMS }}$ has been proposed by [18] as follows:

$$
B_{c} \approx \frac{1}{\alpha \tau_{\mathrm{RMS}}}
$$

where $\alpha$ is a coefficient that depends on the nature of the propagation channel. In work [19], the authors found that $\alpha=1 / 0.15$ in an indoor radio channel.

Figure 8 displays the levels of $\tau_{\mathrm{RMS}}$ as a function of the distance between the $T x$ and each $R x$ when using the two antennas ((a) 3D cubic and (b) dipole). 


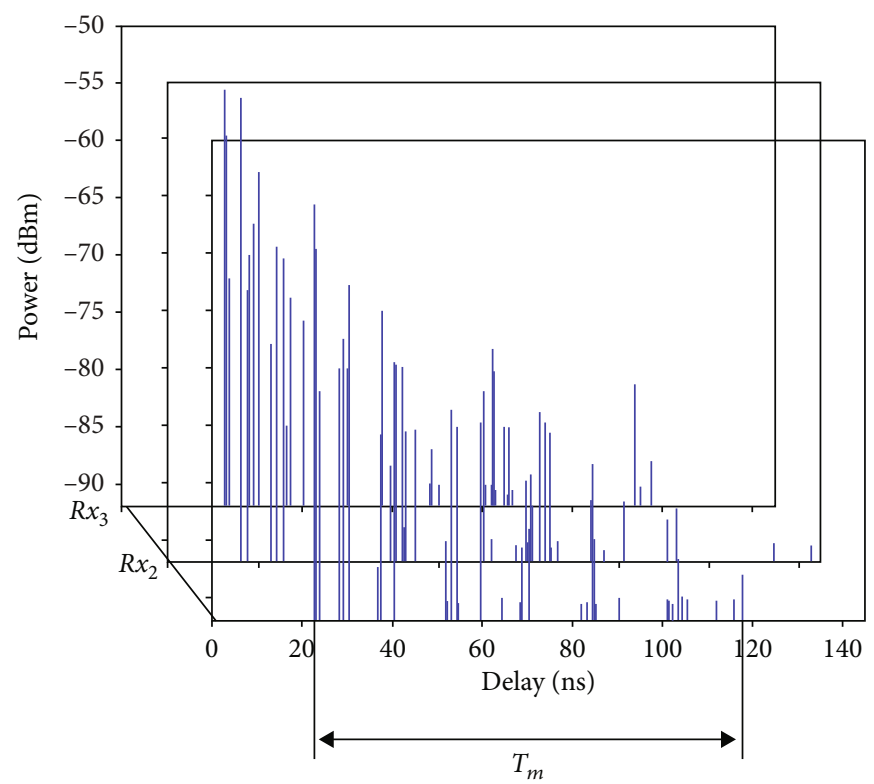

FIGURE 7: Impulse responses (IRs) at the level of three $R x$ using the 3D cubic antenna.

According to the results and when using the $3 \mathrm{D}$ cubic antenna, we have obtained a maximum value of $\tau_{\text {RMS }}$ of $23.66 \mathrm{~ns}$ at a distance $14.03 \mathrm{~m}$. On the other hand, the maximum value of the $\tau_{\mathrm{RMS}}$ obtained when using the conventional dipole antenna is $39.76 \mathrm{~ns}$ at the same distance.

The values of $\tau_{\text {RMS }}$ change depending on the orientation of the antennas as we have shown in the work [8]. Table 3 summarizes the maximum differences between the levels of $\tau_{\text {RMS }}$ obtained during all orientations of the $3 \mathrm{D}$ cubic and dipole antennas in the LOS, NLOS, and OLOS scenarios.

According to the table, the two maximum differences of $\tau_{\text {RMS }}$ obtained when orienting the $3 \mathrm{D}$ cube antenna and the dipole antenna are $9.34 \mathrm{~ns}$ and $28.49 \mathrm{~ns}$, respectively. Therefore, the maximum value of $\tau_{\mathrm{RMS}}$ obtained when using the $3 \mathrm{D}$ cube antenna becomes $33 \mathrm{~ns}$ and when using the dipole antenna becomes $68.25 \mathrm{~ns}$.

Then, the minimum value of $B_{c}$ obtained using the $3 \mathrm{D}$ cubic antenna and the conventional dipole antenna are 4.55 $\mathrm{MHz}$ and $2.2 \mathrm{MHz}$, respectively. Therefore, the signal bandwidth $B_{s}$ must be lower than the coherence band $B_{c}$ in order to limit intersymbol interference (ISI).

For digital modulation, the $B_{s}$ is calculated from the symbol duration $T_{s}$ by

$$
B_{s}=\frac{1}{T_{s}} .
$$

So, to limit ISI, the $T_{s}$ duration must be greater than $219.78 \mathrm{~ns}$ when using the $3 \mathrm{D}$ cubic antenna. On the other hand, the $T_{s}$ duration must be greater than $454.55 \mathrm{~ns}$ when using the conventional dipole antenna.

3.3. Coherence Time. To limit symbol estimation errors (SEE), the duration $T_{s}$ must be less than the coherence time $T_{c}$ resulting from the mobility of network nodes and obstacles. In our case, the nodes are fixed, and the obstacles that are always moving are humans. The influence of obstacle mobility is very negligible compared to that of node mobility.

Consequently, we can consider that the human wears nodes, in order to know only the maximum value of the duration $T_{s}$ that we will not exceed. From Equation (7), we can plot the variation of $T_{c}$ (see Figure 9) as a function of the human speed $V$, which normally does not exceed $1 \mathrm{~m} / \mathrm{s}$.

$$
T_{c} \approx \frac{9}{16 \pi f(V / C)} .
$$

According to the results, the minimum $T_{c}$ is $6.52 \mathrm{~ms}$, which implies that the duration $T_{s}$ must be less than this value in order to limit SEE.

To conclude, the duration $T_{s}$ must be between $219.78 \mathrm{~ns}$ and $6.52 \mathrm{~ms}$ to limit ISI and EES during the use of the 3D cubic antenna. On the other hand, it must be between $454.55 \mathrm{~ns}$ and $6.52 \mathrm{~ms}$ when using the conventional dipole antenna.

The limit of duration $T_{s}$ also limits the bit rate $D$ because $D$ is the product of the inverse of $T_{s}$ (modulation rate $R$ ) and the logarithm to the base 2 of the number of possible states of a symbol (valence $V$ ) [20]:

$$
D=\frac{1}{T_{s}} \log _{2}(V) .
$$

Tables 4 and 5 list the limiting values of $D$ as a function of $V$ when using the 3D cubic antenna and the conventional dipole antenna, respectively.

Usually, the bit rate $D$ must be high to ensure low data transfer time. Therefore, the duration $T_{s}$ must be chosen close to the minimum value. This implies that we will not have SEE whatever the movement of nodes and obstacles. 


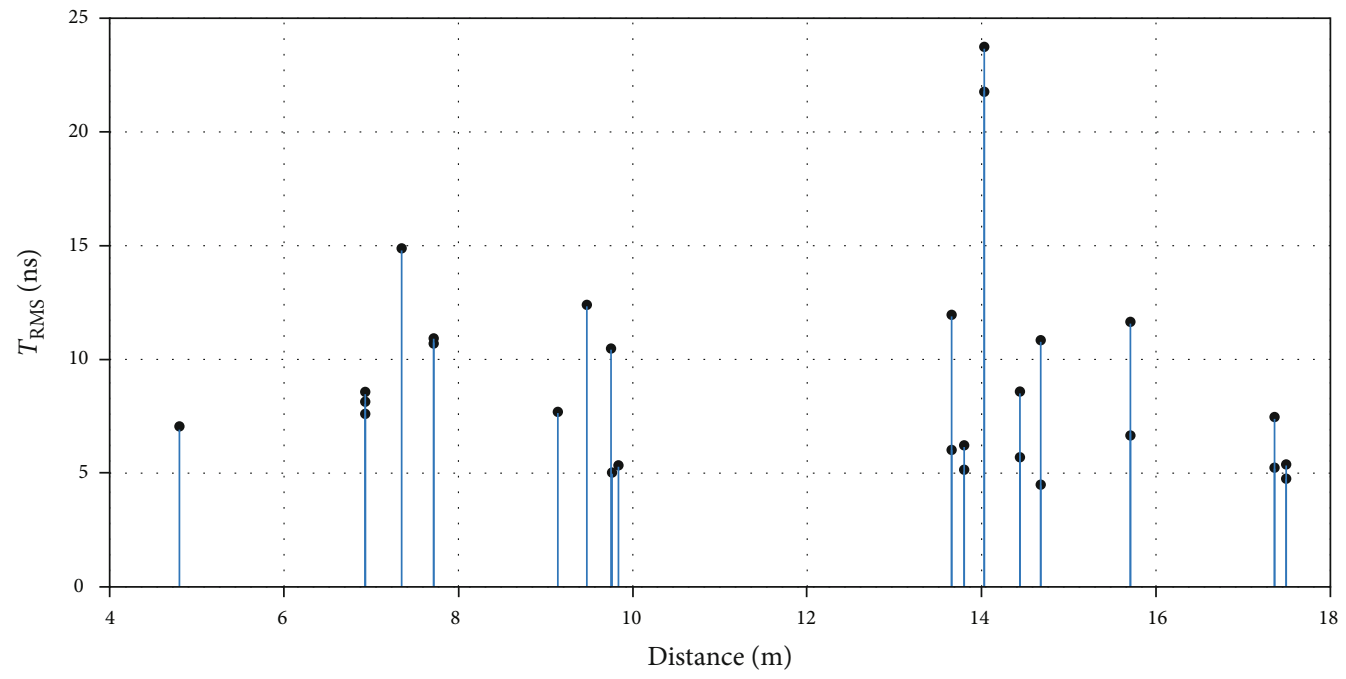

(a)

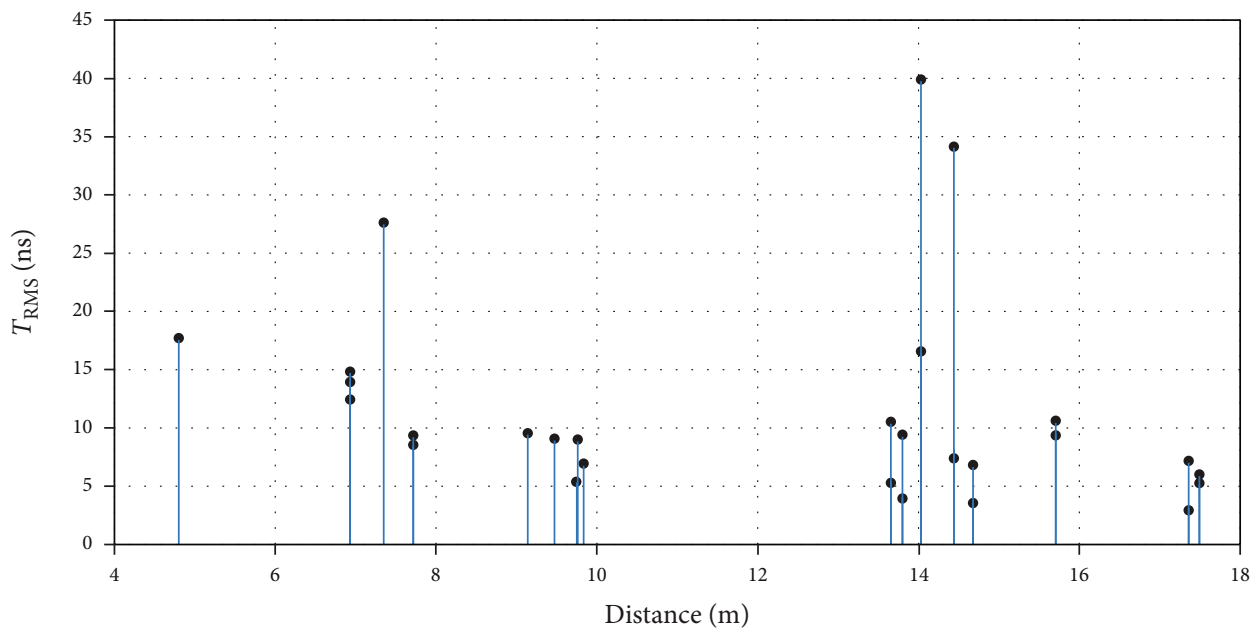

(b)

FIgURE 8: Dispersion of delays $\tau_{\text {RMS }}$ as a function of the distance between $T x$ and each $R x$ using the antenna: (a) 3D cubic and (b) dipole.

TABle 3: Maximum deviations between the levels of $\tau_{\text {RMS }}$ during the orientations of the 3D cubic and dipole antennas [8].

\begin{tabular}{lcc}
\hline Scenarios & 3D cubic antenna & Dipole antenna \\
\hline LOS & $6.48 \mathrm{~ns}$ & $28.49 \mathrm{~ns}$ \\
NLOS & $4.64 \mathrm{~ns}$ & $23.62 \mathrm{~ns}$ \\
OLOS & $9.34 \mathrm{~ns}$ & $27.97 \mathrm{~ns}$ \\
\hline
\end{tabular}

From both Tables 4 and 5, we have a very high bit rate when using the $3 \mathrm{D}$ cubic antenna compared to using the conventional dipole antenna. Then, the use of our 3D cubic antenna is better than the conventional dipole antenna.

\section{Conclusions}

In this work, we have modeled a building that has been selected as a propagation channel where the RSN network is deployed for control and monitoring. For the transmission, we have used the $3 \mathrm{D}$ cubic antenna that we have designed and the conventional dipole antenna which is the most used in the RSN network. By means of the 3D ray tracing method, we have predicted the characteristics of the propagation channel in order to improve the communication between the devices of the RSN network in terms of the appropriate transmission parameters such as the transmission power $P_{t}$ and the duration of a symbol $T_{s}$. We have found as results to fight against fading of the received signal that a transmission power of $0 \mathrm{dBm}$ is sufficient when using our $3 \mathrm{D}$ cube antenna. However, it is necessary to increase this power during the use of the conventional dipole antenna, which automatically leads to a decrease in the lifetime of the devices, which is a disadvantage. To limit ISI and EES, the symbol duration must be close to a minimum value of the order of $219.78 \mathrm{~ns}$ when using our 3D cubic antenna and of the order of $454.55 \mathrm{~ns}$ when using the conventional dipole antenna. These two values result in different bit rates. The use of our 


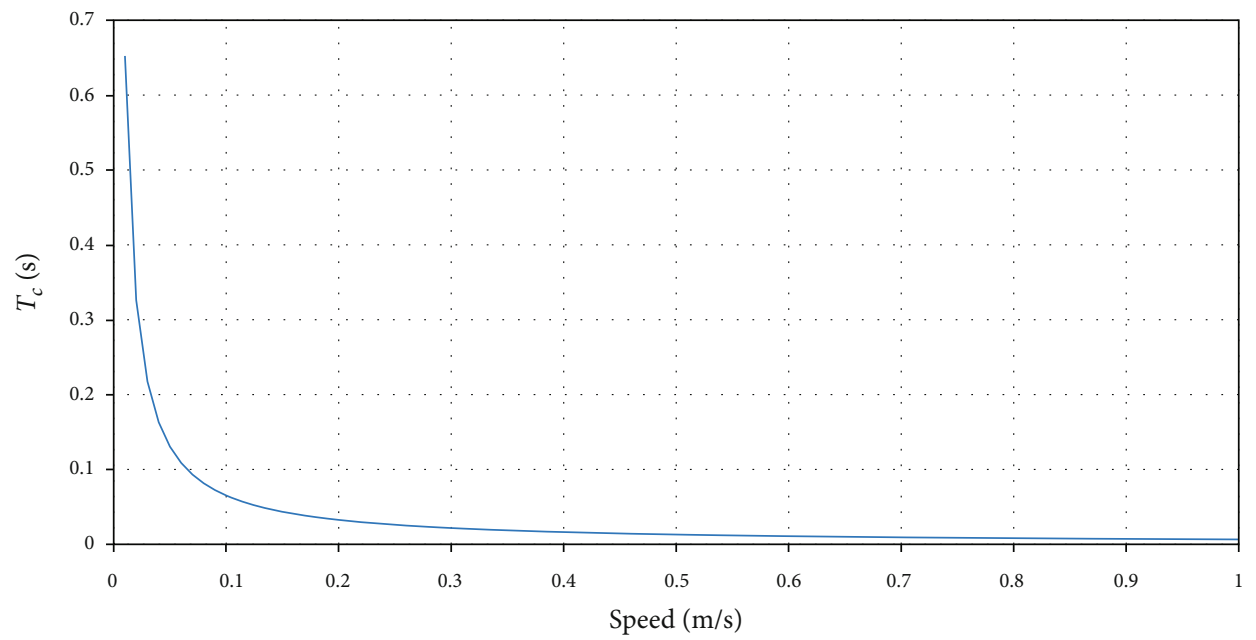

Figure 9: Coherence time $T_{c}$ as a function of speed $V$.

TABLE 4: Limiting values of bit rate $D$ as a function of valence $V$ when using the $3 \mathrm{D}$ cubic antenna.

\begin{tabular}{lcc}
\hline Minimum value & Parameters & Maximum value \\
\hline $153.37 \mathrm{bit} / \mathrm{s}<$ & $D(V=2)$ & $<4.55 \mathrm{Mbit} / \mathrm{s}$ \\
$306.75 \mathrm{bit} / \mathrm{s}<$ & $D(V=4)$ & $<9.1 \mathrm{Mbit} / \mathrm{s}$ \\
$460.12 \mathrm{bit} / \mathrm{s}<$ & $D(V=8)$ & $<13.65 \mathrm{Mbit} / \mathrm{s}$ \\
\hline
\end{tabular}

TABLE 5: Limiting values of bit rate $D$ as a function of valence $V$ when using the conventional dipole antenna.

\begin{tabular}{lcc}
\hline Minimum value & Parameters & Maximum value \\
\hline $153.37 \mathrm{bit} / \mathrm{s}<$ & $D(V=2)$ & $<2.2 \mathrm{Kbit} / \mathrm{s}$ \\
$306.75 \mathrm{bit} / \mathrm{s}<$ & $D(V=4)$ & $<4.4 \mathrm{Mbit} / \mathrm{s}$ \\
$460.12 \mathrm{bit} / \mathrm{s}<$ & $D(V=8)$ & $<6.6 \mathrm{Mbit} / \mathrm{s}$ \\
\hline
\end{tabular}

antenna provides a high bit rate in order to ensure a low data transfer time. To conclude, our antenna gives several advantages for the RFID sensor array compared to the most commonly used antennas.

\section{Data Availability}

Requests for access to data should be made to Abdelhamid BOU-EL-HARMEL,

abdelhamid.bouelharmel@usmba.ac.ma.

\section{Conflicts of Interest}

The authors declare that there is no conflict of interest regarding the publication of this paper.

\section{References}

[1] S. Mejjaouli and R. F. Babiceanu, "RFID-wireless sensor networks integration: decision models and optimization of logistics systems operations," Journal of Manufacturing Systems, vol. 35, pp. 234-245, 2015.
[2] A. Mitrokotsa and C. Douligeris, "Integrated RFID and sensor networks: architectures and applications," RFID and Sensor Networks: Architectures, Protocols, Security and Integrations, vol. 512, pp. 511-535, 2009.

[3] R. C. Hadarig, M. E. de Cos, and F. Las-Heras, "UHF dipole-AMC combination for RFID applications," IEEE Antennas and Wireless Propagation Letters, vol. 12, pp. 1041-1044, 2013.

[4] A. E. Abdulhadi and R. Abhari, "Design and experimental evaluation of miniaturized monopole UHF RFID tag antennas," IEEE Antennas and Wireless Propagation Letters, vol. 11, pp. 248-251, 2012.

[5] A. Bou-El-Harmel, A. Benbassou, and J. Belkadid, "Design of a three-dimensional antenna UHF in the form cubic intended for RFID, wireless sensor networks (WSNs) and RFID sensor networks (RSNs) applications," International Journal on Communications Antenna and Propagation (IRECAP), vol. 4, no. 6, pp. 260-264, 2014.

[6] A. Bou-El-Harmel, A. Benbassou, and J. Belkadid, "Optimization of a 3D UHF cubic antenna with quasi-isotropic radiation pattern for RFID, WSN and RSN applications," WSEAS Transactions on Communications, vol. 14, pp. 365-373, 2015.

[7] A. Bou-El-Harmel, A. Benbassou, and J. Belkadid, "Design and development of a new electrically small 3D UHF spherical antenna with $360^{\circ}$ of opening angle in the whole space for RFID, WSN, and RSN applications," International Journal of Antennas and Propagation, vol. 2016, Article ID 2906149, 18 pages, 2016.

[8] A. Bou-El-Harmel, A. Benbassou, J. Belkadid, and N. Mechatte, "Effect of quasi-isotropic antenna orientation on indoor multipath propagation characteristics in RSN applications," International Journal of Antennas and Propagation, vol. 2017, Article ID 2686123, 13 pages, 2017.

[9] "La cité de l'innovation," http://www.usmba.ac.ma/ citt/.

[10] C.-F. Yang, C.-J. Ko, and B.-C. Wu, "A free space approach for extracting the equivalent dielectric constants of the walls in buildings," in IEEE Antennas and Propagation Society International Symposium, pp. 1036-1039, Baltimore, MD, USA, July 1996.

[11] O. N. Stavroudis, The Mathematics of Geometrical and Physical Optics, John Wiley \& Sons, 2006. 
[12] J. Russell and R. Cohn, Uniform Theory of Diffraction, Book on Demand, 2012.

[13] L. Aveneau, Y. Pousset, R. Vauzelle, and M. Mériaux, "Development and evaluations of physical and computer optimizations for the 3D UTD model," in AP2000 Millennium Conference on Antennas \& Propagation, pp. 9-14, Davos, Switzerland, 2000.

[14] G. E. Athanasiadou and A. R. Nix, "A novel 3-d indoor raytracing propagation model: the path generator and evaluation of narrow-band and wide-band predictions," IEEE Transaction on Vehicular Technology, vol. 49, no. 4, pp. 1152-1168, 2000.

[15] Z.-Y. Liu, L.-X. Guo, and X. Meng, "Efficient threedimensional ray-tracing model for electromagnetic propagation prediction in complex indoor environments," The Journal of the Optical Society of America A, vol. 30, no. 8, p. 1654, 2013.

[16] A. Bildea, Link Quality in Wireless Sensor Networks, HAL, 2015.

[17] Analog Devices, Analog Devices Wireless Sensor Network (WSN) Solutions, Analog devices, 2014, Project Code: APMWSN-2014.

[18] M. J. Gans, "A power-spectral theory of propagation in the mobile-radio environment," IEEE Transactions on Vehicular Technology, vol. 21, no. 1, pp. 27-38, 1972.

[19] K. Pahlavan and S. J. Howard, "Frequency domain measurements of indoor radio channels," Electronics Letters, vol. 25, no. 24, pp. 1645-1647, 1989.

[20] D. Dromard and D. Seret, Architecture des réseaux, Pearson Education France, 2013. 